\title{
CONTROL OF A PORTFOLIO OF INSURANCE CONTRACTS
}

\section{KARL BORCH}

Bergen

\section{Introduction}

1.1. In this paper we shall consider a given portfolio of insurance contracts, and we shall study the following two problems:

(i) How should this portfolio be reinsured?

(ii) What reserves should the company maintain to pay claims which will be made under the contracts in the portfolio?

This means that we shall ignore all questions as to how the company acquired the portfolio, i.e. some of the most important questions concerning management control of insurance companies, such as rating policy, underwriting control etc.

1.2. Even the two simple problems, which are singled out for study in this paper, cannot be solved unless we specify the objectives and the external circumstances of the company.

It is obvious that the reinsurance problem cannot be solved unless we know something about the company's "attitude to risk", and about the cost of obtaining cover for various kinds of risk in the reinsurance market.

It is also obvious that we cannot solve the reserve problem unless we specify the safety requirements, which the company has to satisfy. We shall see in the following that we may also have to specify the portfolios, which the company expects to underwrite in the future.

\section{The Basic Model}

2.1. We shall now consider an insurance company, which has underwritten a portfolio of short-term insurance contracts. We shall assume:

(i) $F(x)=$ the probability that claim, payments under these contracts shall not exceed $x$. 
(ii) $P=$ the amount of premiums, which the company collected by underwriting the portfolio.

(iii) $S=$ the initial capital of the company.

When all contracts in the portfolio have expired, the company will hold a capital

$$
y=S+P-x
$$

It is clear that $y$ is a stochastic variable with the distribution

$$
G_{0}(y)=F(S+P--y) \quad(y \leq S+P)
$$

It is convenient to refer to $G_{0}(y)$ as the profit distribution associated with the portfolio.

2.2. By reinsurance arrangements it may be possible for the company to change the profit distribution.

Let us assume that the company by suitable reinsurance arrangements can obtain any of the profit distributions $G_{1}(y) \ldots G_{n}(y) \ldots$ The problem is then to determine the "best" among these attainable distributions. In order to solve this problem, we must know the company's preference ordering over the set of attainable profit distributions. Such preference orderings have been discussed in other papers [2] and [3]. It has been shown that if the ordering is consistent, there exists a function $u(x)$ which represents the ordering in the sense that

$$
\int_{-\infty}^{+\infty} u(y) d G_{i}(y)>\int_{-\infty}^{+\infty} u(y) d G_{j}(y)
$$

if and only if the profit distribution $G_{i}(y)$ is preferred to $G_{j}(y)$.

This result, due to von Neumann and Morgenstern [5], gives us an attractive operational formulation of the reinsurance problem. However, this formulation is useful only if we know something about the shape of the utility function $u(x)$, which represents the preference ordering. We shall see in the following that we can obtain some information about this function by placing our, essentially static, problem in its natural dynamic setting.

2.3. In our model there is a probability

$$
\mathrm{I}-F(S+P)
$$

that the company shall be unable to pay the claims, which are made under the contracts in the portfolio. 
Let us now assume that somebody-the company's management or the Government inspector-decides that the company cannot operate unless this probability is smaller than $\alpha$.

This means that if the requirement is not satisfied, i.e. if

$$
\mathbf{I}-F(S+P)>\alpha
$$

the company must either obtain additional capital, or seek a reinsurance arrangement, so that the condition is met.

If, on the other hand,

$$
\mathrm{I}-F(S+P) \leq \alpha
$$

there is no obvious reason why the company should risk all its capital in the insurance business. It is natural to assume that the company in this case will pay a dividend $s$, determined by the equation

$$
\mathrm{I}-F(S+P-s)=\alpha
$$

This gives us a solution to the reserve problem, provided that $\alpha$ is given, for instance by regulations imposed by the Government. If there are no such regulations, the company can pay out all its assets as dividend, and calmly face certain ruin. However, experience tells us that insurance companies do not behave in this way. All available evidence indicates that they want to survive and stay in business. This observation naturally leads us to consider dynamic models.

2.4. Let us now assume that our company in each successive period receives a premium $P$ by underwriting a portfolio with a claim distribution $F(x)$. Let further $S$ be the initial capital of the company.

We shall then tentatively assume that the company considers $Z$ as a sufficient reserve for the portfolio in question, i.e. whenever its capital exceeds $Z$, the excess will be paid out as dividend. This means of course that the acceptable ruin probability is $\alpha=\mathrm{I}$ $F(Z+P)$.

Let now $s_{t}$ be the dividend paid after the $t^{\prime}$ th underwriting period, and consider the expected discounted value of the sequence of dividend payments:

$$
V(S, Z)=E\left\{\sum_{t=0}^{\infty} v^{t} s_{t}\right\}
$$


It is easy to see that $V(S, Z)$ must satisfy the integral equation

$$
V(S, Z)=v \int_{0}^{s+P} V(S+P-x, Z) d F(x)
$$

for $0 \leq S \leq Z$

By definition we have

$$
V(S, Z)=S-Z+V(Z, Z) \text { for } S>Z
$$

If we assume that the company has to go out of business if it loses all its capital, we have

$$
V(S, Z)=0 \quad \text { for } S<0
$$

2.5. It is possible to solve the integral equation in the preceding paragraph by classical means. The solution will, however, be exceedingly complex, and we shall not attempt to discuss the problem in its full generality.

It is not surprising that our model leads to rather formidable mathematical problems, since it is direct generalization of the collective risk theory. In fact, our model reduces to that of Lundberg if we let $Z$ go to infinity, i.e. if we assume that the company never considers any dividend payment.

2.2. To get some idea about the nature of the solution, we shall consider the special case:

$$
F(x)=\mathrm{I}-e^{-x}
$$

The integral equation can then be written

$$
V(S, Z)=v e^{-S-P} \int_{0}^{S+P} V(x, Z) e^{x} d x \quad(S<Z)
$$

or by omitting $\cdot Z$ for simplicity

$$
V(S)=v e^{-S-P} \int_{0}^{S+P} V(x) e^{x} d x
$$

Differentiation with respect to $S$ gives

$$
V^{\prime}(S)=v V(S+P)-v e^{-S-P} \int_{0}^{S+P} V(x) e^{x} d x
$$

Adding this to the original equation, we obtain the differentialdifference equation

$$
V^{\prime}(S)+V(S)-v V(S+P)=o
$$


It is easy to see that this equation has a solution of the form

$$
V(S)=e^{\alpha S}
$$

where $\alpha$ satisfies the characteristic equation

$$
\alpha+\mathrm{I}-v e^{\alpha P}=0
$$

This equation has infinitely many roots, which all are simple. This means that the general solution of our differential-difference equation is of the form

$$
V(S)=\sum_{r} c_{r} e^{\alpha_{r} S}
$$

where the sum is taken over all roots of the characteristic equation. $c_{r}$ are constants, which must be determined so that the "boundary conditions"

$$
\begin{array}{ll}
V(S, Z)=S-Z+V(Z, Z) & S>0 \\
V(S, Z)=0 & S<0
\end{array}
$$

are satisfied.

We shall not pursue this subject any further, since it has been discussed in considerable detail in the literature, i.a. in a recent book by Bellman and Cooke [I]. Instead we shall study an even simpler example.

\section{The Simplest Possible Case}

3.1. We shall now consider an insurance company which in each operating period receives a premium of $I$ by underwriting a porttolio of insurance contracts, which can lead to the claim payments.

$$
\begin{array}{ll}
\text { Either } & 0 \text { with probability } p \\
\text { or } & 2 \text { with probability } q=\mathrm{I}-p
\end{array}
$$

This means that the company in each period engages in a gamble, which can lead to either a loss or a gain of one unit. We shall assume that $p>q$, i.e. that the gambles are favorable to the company.

We shall further assume that the company has an initial capital $S$, and that the game will terminate if the capital becomes negative, i.e. that the company will have to go out of business if it becomes insolvent.

If the sole objective of the company is to stay in business as 
long as possible, it is obvious that the company will never pay any dividend.

In order to obtain a non-trivial model, we shall assume that the company wants to pay dividends, and that its overall objective is to maximize the expected discounted value of the dividends, which will be paid during the company's lifetime.

3.2. The model which we have outlined was first studied by Shubik and Thompson [6], and it has been discussed in some detail in another paper [4].

Shubik and Thompson proved that the optimal dividend policy is to let the capital increase up to an amount $Z$, and then pay out any excess as dividend immediately.

Let now $w_{n}(S, Z)$ be the probability that the first dividend shall be paid after $n$ periods, provided that:

$S=$ the initial capital of the company

$Z=$ the capital which the company decides to accumulate before any dividend is paid.

At this stage of our analysis there is no loss of generality if we assume that $S$ and $Z$ are integers.

It is easy to see that this probability must satisfy the recurrence relation

$$
w_{n+1}(S, Z)=p w_{n}(S+\mathrm{I}, Z)+q w_{n}(S-\mathrm{I}, Z)
$$

subject to the boundary conditions

$$
\begin{array}{ll}
w_{0}(S, Z)=0 & \text { for } S \leq Z \\
w_{0}(S, Z)=\mathrm{I} & \text { for } S>Z \\
w_{n}(S, Z)=0 & \text { for } S>Z \text { and } n>0 . \\
w_{n}(S, Z)=0 & \text { for } S<0
\end{array}
$$

3.3. Let us now introduce the generating function

$$
W(S, Z)=\sum_{n=0}^{\infty} v^{n} w_{n}(S, Z)
$$

It is easy to see that this function must satisfy the difference equation

$$
W(S, Z)=p v W(S+\mathrm{I}, Z)+q v W(S-\mathrm{I}, Z)
$$

The boundary conditions are easily established. Taking these 
into account, we find that the difference equation has the solution

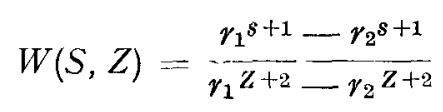

where $r_{1}$ and $r_{2}$ are the roots of the characteristic equation

$$
r=p v r^{2}+q v
$$

If the variable $v$ is interpreteted as a discount factor, $W(S, Z)$ will be the expected discounted value of the first unit paid as dividend.

3.4. Let now, as in para 2.4., $V(S, Z)$ stand for the expected discounted value of the dividends which our company will pay during its lifetime.

Since we have assumed that $S$ and $Z$ are integers, the first dividend will be $\mathrm{I}$, and it will be paid when the company's capital reaches $Z+\mathrm{I}$. When this dividend has been paid, the company will enter the next operating period with a capital $Z$.

From these considerations it follows that for $0 \leq S \leq Z$

$$
V(S, Z)=\{\mathrm{x}+V(Z, Z)\} W(S, Z)
$$

By putting $S=Z$, we obtain

and

$$
V(Z, Z)=\frac{W(Z, Z)}{\mathrm{I}-W(Z, Z)}
$$

or

$$
V(S, Z)=\frac{W(S, Z)}{\mathrm{I}-W(Z, Z)}
$$

$$
V(S, Z)=\frac{r_{1}{ }^{S+1}-r_{2} S+1}{\left(r_{1} Z+2-r_{2}^{Z+2}\right)-\left(r_{1}^{Z+1}-r_{2}^{Z+1}\right)}
$$

It is easy to see that there is a unique value of $Z$, independent of $S$, which will maximize $V(S, Z)$. However, some caution is required, since we so far have assumed that $S$ and $Z$ both are integers.

For $S>Z$ we have by our definition of the dividend policy

$$
V(S, Z)=S-Z+V(Z, Z)
$$

and for $S<0$ we have obviously

$$
V(S, Z)=0
$$


3.5. It may be useful to illustrate these results by a simple numerical example. For this purpose we shall take $r_{1}=$ I.I and $r_{2}=0.7$. These values correspond approximately to

$$
\mathrm{p}=0.565, \quad q=0.435 \text { and } v=0.983
$$

Table I gives the value of $V(S, Z)$ for some selected values of $S$ and $Z$.

TABLE I

$V(S, Z)=$ Expected discounted value of dividend payments

$Z=$ Capital required before dividends can be paid

\begin{tabular}{cccccccc}
\hline $\begin{array}{c}S=\text { Initial } \\
\text { Capital }\end{array}$ & o & I & 2 & 3 & 4 & 5 & 6 \\
\hline o & I.25 & I.49 & I.70 & I.83 & I.89 & I.89 & I.82 \\
I & 2.25 & 2.69 & 3.05 & 3.30 & 3.40 & 3.40 & 3.27 \\
2 & 3.25 & 3.69 & 4.19 & 4.52 & 4.67 & 4.67 & 4.49 \\
3 & 4.25 & 4.69 & 5.19 & 5.56 & 5.79 & 5.79 & 5.56 \\
4 & 5.25 & 5.69 & 6.19 & 6.56 & 6.81 & 6.82 & 6.55 \\
5 & 6.25 & 6.69 & 7.19 & 7.56 & 7.81 & 7.83 & 7.50 \\
\hline
\end{tabular}

We see that in this example the optimal dividend policy is given by $Z=5$, at least as long as we only admit integral values of $Z$. To complete our analysis we should of course investigate the meaning of nonintegral values of $Z$. We shall, however, ignore this problem since it is not very relevant to the main purpose of the present paper.

3.6. We have so far indicated how the reserve problem can be solved for a given portfolio. In order to do this, we had to make assumptions about the portfolios, which the company expects to underwrite in the future-i.e. to examine the static problem in a dynamic setting.

In order to attack the reinsurance problem, let us first assume that our insurance company has adopted an optimal dividend policy, corresponding to a certain integral number $Z$.

If the company at the beginning of a period holds a capital $S<Z$, the expected discounted value of its future dividend payments is given by

$$
V(S, Z)=K\left\{r_{1} S+1-r_{2} S+1\right\}
$$

where $K$ is independent of $S$. 
Let us further assume that at this point the company is offered an insurance contract of the type

Gain $R_{1}$ with probability $\alpha$

Loss $R_{2}$ with probability $\mathrm{I}-\alpha$

We can think of this as an invitation to provide short-term reinsurance cover for another company.

If our company accepts the offer, the expected value of its future dividend payments will become

$$
\alpha V\left(S+R_{1}, Z\right)+(\mathrm{I}-\alpha) V\left(S-R_{2}, Z\right)
$$

If the company maintains the overall objective of maximizing the expected discounted value of the dividends which will be paid during its whole lifetime, it will accept the offer if and only if

$$
\alpha V\left(S+R_{\mathbf{1}}, Z\right)+(\mathrm{I}-\alpha) V\left(S-R_{2}, Z\right)>V(S, Z)
$$

This means, however, that the company will make its decision as if it wanted to maximize expected utility, with $V(S, Z)$ serving as the utility function.

In some earlier papers [2] and [3] it has been pointed out that a rational theory of reinsurance must be based on a utility concept. This is, however, not a very useful result, unless we know something about the shape of the utility function, which the company seeks to maximize. The considerations above show that this function can be taken as determined by the long-term objectives of the company-at least when the company acts as reinsurer.

3.3. Before we analyse the company's own reinsurance arrangements, we should note that the expression which we found for $V(S, Z)$ in para 3.4. is valid only for integral values of $S$ and $Z$. If we want to interpret $V(S, Z)$ as a utility function, it is necessary to define it also for non-integral values of $S$.

If $S$ and $Z$ are integers, we have:

(i) The first dividend cannot be paid earlier than after $Z-S+$ I periods, and this dividend will necessarily be equal to $\mathrm{I}$.

(ii) The company can be forced out of business at the earliest after $S+$ I periods.

Let now $[S]$ be the largest integer not exceeding $S$. If $S$ is not an integer, we have, assuming that $Z$ is an integer 
(i) The first dividend will be equal to $S-[S]$, and it can be paid at the earliest after $Z-[S]$ periods

(ii) Ruin can at the earliest occur after $[S]+$ I periods.

With these observations we can just repeat the argument used in paras 3.3. and 3.4.

When $S$ is not an integer, we have to substitute [S] and $Z-\mathbf{I}$ for $S$ and $Z$, hence the generating function will be

$$
W(S, Z)=W([S], Z-\mathrm{I})
$$

and we have, since the first dividend will be $S-[S]$ :

$$
V(S, Z)=\{S-[S]+V(Z, Z)\} W([S], Z-\mathbf{I})
$$

This expression can be written

$$
V(S, Z)=\{S-[S]\} \frac{r_{1}[S+1]-r_{2}{ }^{[S+1]}}{r_{1}^{Z+1}-r_{2} Z+1}+V([S], Z)
$$

and it is easy to verify that it holds also for integral values of $S$.

From this we see that the function $V(S, Z)$ has jumps for integral values of $S$. Between the jumps $V(S, Z)$ will increase linearly with $S$.

Table II below gives the value of $V(S, 5)$ for some selected values of $S$ for the numerical example introduced in para 3.5 .

TABLE II

$V(S, 5)=$ Expected discounted value of dividend payments for an optimal dividend policy

\begin{tabular}{llll}
\hline$S$ & $V(S, 5)$ & $S$ & $V(S, 5)$ \\
\hline o & I.89 & I.75 & 3.73 \\
0.25 & 1.95 & 2.00 & 4.67 \\
0.50 & 2.01 & 2.25 & 4.82 \\
0.75 & 2.07 & 2.50 & 4.97 \\
I.00 & 3.40 & 2.75 & 5.12 \\
I.25 & $3.5 \mathrm{I}$ & 3.00 & 5.79 \\
$\mathrm{I} .5^{\circ}$ & 3.62 & 3.25 & 5.97 \\
\hline
\end{tabular}

3.8. It is obvious that our insurance company-acting as reinsurer - can make some decisions, which will seem very peculiar to an outside observer. Let us as an illustration assume that the company's capital is $S=\mathbf{I} .75$. If the company has adopted the optimal 
dividend policy, defined by $Z=5$, the expected discounted value of future dividend payments will be

$$
V(\mathrm{r} .75,5)=3.73
$$

Let us now assume that the company is offered a premium of 0.5 if it will cover a risk with the claim distribution

$$
\begin{array}{ll}
\text { o with probability } & 0.33 \\
\text { I with probability } & 0.67
\end{array}
$$

Any actuary worthy of the name will advise against accepting this offer. If, however, the company accepts the offer in spite of actuarial orthodoxy, the expected discounted value of the future dividend payments will increase to

$$
0.33 V(2.25,5)+0.67 V(\mathbf{1} .25,5)=3.94
$$

Hence it is to the advantage of the company to accept this offer, even if it is grossly unfair.

If we want to justify this paradox, we would have to argue that the company is uncomfortably close to ruin. Hence it appears worth while to take a chance-against unfavorable odds-to get out of the danger zone. It may, however, not be easy to sell this argument to the General Manager of the company.

3.9. To illustrate the effect of reinsurance, let us assume that the company reinsures a quota share $\mathrm{I}-k$ of its portfolio on "original terms", i.e. the company retains a quota $k$. We shall assume that this arrangement is made for one underwriting period only, i.e. we exclude any long-term reinsurance treaties. Let $V(S, Z, k)$ be the expected discounted value of future dividend payments under this short-term reinsurance arrangement. Our problem is then to determine

$$
\operatorname{Max}_{0<k<1}(V(S, Z, k))
$$

It is easy to see that we have

$$
V(S, Z, k)=v p V(S+k, Z)+v q V(S-k, Z)
$$

and for the two extremes:

$$
\begin{aligned}
& V(S, Z, \mathrm{I})=V(S, Z) \\
& V(S, Z, \mathrm{o})=v V(S, Z)
\end{aligned}
$$


Since $V(S, Z)$ is piece-wise linear, the maximum must occur, either for $k=\mathrm{I}$, or at one of the jumps.

From this it follows that the optimal retention is:

$$
\begin{array}{ll}
k=\mathbf{I} & \text { for } S-[S] \leq \frac{1}{2} \\
k=S-[S] & \text { for } S-[S]>\frac{1}{2}
\end{array}
$$

Intuitively this means that the company should reinsure to avoid the possibility of a downward jump in $V(S, Z)$, if this can be done without losing a possibility of an upward jump. This kind of solution is sometimes referred to as "bang-bang control".

\section{Concluding Remarks}

4.1. In the preceding paragraphs we have tried to formulate the control problems of insurance companies in an operational manner, and we have indicated how these problems can be solved. It appears that this formulation of the problems leads to a generalization of the collective risk theory, created by F. Lundberg. It also appears that fairly advanced mathematical tools will be required to analyse these problems in full generality. The author expects to discuss this subject in more detail in a later paper.

4.2. Some of the results indicated in this preliminary paper, may appear paradoxal. It is worth noting that this is not due to the extreme simplicity of the model which we have studied. The paradoxes are caused by the discontinuities of the function $V(S, Z)$, and such discontinuities will occur also in more general models. The basic integral equation in para 2.4 may in general only have a piece-wise continuous solution for $S<Z-P$. From this it follows that an insurance company occasionally should accept an actuarially unfair contract-if the company seeks to maximize the expected, discounted value of its dividend payments.

Los Angeles, April I965

\section{REFERENCES}

[I] Bellman, R. and Cooke, K. L.: Differential-Difference Equations, Academic Press 1963.

[2] Borch, K.: "The Utility Concept applied to the Theory of Insurance", The ASTIN Bulletin, Vol. I, pp. 245-255.

[3] Borch, K.: "The Objectives of an Insurance Company", Skandinavisk Aktuarietidskrift, 1962, pp. I62-I 75 . 
[4] Borch, K.: "The Optimal Management Policy of an Insurance Company" Proceedings of the Casualty Actuarial Society, Vol. 5I. pp. 182-197.

[5] Neumann, J. von and Morgenstern, O., Theory of Games and Economic Behavior, 2nd Edition, Princeton, 1947.

[6] Shubik, M. and Thompson, G., "Games of Economic Survival", Naval Research Logistics Quarterly, 1959, pp. II I-I23. 